\title{
A new method of measuring electric dipole moments in storage rings
}

\author{
F.J.M. Farley ${ }^{7}$, K. Jungmann ${ }^{4}$, J.P. Miller², W.M. Morse ${ }^{3}$, Y.F. Orlov ${ }^{5}$, \\ B.L. Roberts ${ }^{2}$, Y.K. Semertzidis ${ }^{3}$, A. Silenko ${ }^{1}$, E.J. Stephenson 6 \\ ${ }^{1}$ Belarusian State University, Belarus; ${ }^{2}$ Physics Department, Boston University, Boston, MA 02215; \\ ${ }^{3}$ Brookhaven National Laboratory, Upton, NY 11973; ${ }^{4}$ Kernfysisch Versneller Instituut, Groningen; \\ ${ }^{5}$ Newman Laboratory, Cornell University, Ithaca, NY 14853; ${ }^{6}$ IUCF, Indiana University, Bloomington, IN 47408 ; \\ ${ }^{7}$ Department of Physics, Yale University, New Haven, CT 06520.
}

(Dated: November 19, 2018)

\begin{abstract}
A new highly sensitive method of looking for electric dipole moments of charged particles in storage rings is described. The major systematic errors inherent in the method are addressed and ways to minimize them are suggested. It seems possible to measure the muon EDM to levels that test speculative theories beyond the standard model.
\end{abstract}

PACS numbers: 13.40.Em, 12.60.Jv, 14.60.Ef, 29.20.Dh

The existence of a permanent electric dipole moment (EDM) for an elementary particle would violate parity (P) and time reversal symmetry (T) [1]. Therefore under the assumption of CPT invariance, a non-zero EDM would signal $\mathrm{CP}$ violation. In the standard model, the electron EDM is $<10^{-38} \mathrm{e} \cdot \mathrm{cm}[2]$ with the muon EDM scaled up by the mass ratio $m_{\mu} / m_{e}$, a factor of 207 , but some new theories predict much larger values $[3,4]$. For example, ref. [4] predicts the muon EDM could be as large as $5 \times 10^{-23} \mathrm{e} \cdot \mathrm{cm}$, while the electron EDM is predicted to be $\sim 10^{-28} \mathrm{e} \cdot \mathrm{cm}$, an order of magnitude below the present limit [5]. The current $95 \%$ confidence limit for the muon EDM is $10^{-18} \mathrm{e} \cdot \mathrm{cm}[6]$. This paper discusses a new way of using a magnetic storage ring to measure the EDM of the muon, which also can be applied to other charged particles.

To measure the EDM experimentally, the particle should be in an electric field which exerts a torque on the dipole and induces an observable precession of its spin. If the particle is charged this electric field inevitably accelerates the particle; it will move to a region where the field is zero or leave the scene. An example is the nucleus at the center of an atom in equilibrium; the net force and therefore the net electric field at the nucleus must average to zero according to Schiff's theorem 7]. Any applied external electric field will be shielded from the nucleus by the electrons in the atom. The overall effect is to suppress the EDM signal, making it more difficult to measure. The suppression would be total but for the many known exceptions to Schiff's theorem when weak and strong forces, weak electron-nucleon forces, finite particle sizes, and relativistic effects are included. Suppression of the EDM signal by Schiff's theorem is completely avoided in a magnetic storage ring [8, 9] such as proposed here, because the particle is not in equilibrium; there is a net centripetal force, and this force is entirely supplied by a net electric field as seen in the muon rest frame.

In particular, when a muon of velocity $\vec{\beta}=\vec{v} / c$ and relativistic mass factor $\gamma=\left(1-\beta^{2}\right)^{-\frac{1}{2}}$ is circulating in a horizontal plane due to a vertical magnetic field $\vec{B}$, it will according to a Lorentz transformation experience both an electric and a magnetic field, $\vec{E}^{*}$ and $\vec{B}^{*}$, in its own rest frame. The so-called motional electric field, $\vec{E}^{*}=\gamma c \vec{\beta} \times \vec{B}$, can be much larger than any practical applied electric field. Its action on the particle supplies the radial centripetal force, Thomas spin precession, and spin precession due to any non-vanishing EDM. $\vec{B}^{*}$ produces precession due to the muon magnetic moment. The combined spin precession due to the Thomas precession and torque on the magnetic moment is vertically directed and is given 10, 11], in terms of the laboratory frame field, by $\omega_{a}=a(e B / m)$, where $a=(g-2) / 2$ is the magnetic anomaly. This is referred to as the " $(g-2)$ " precession, and if it were acting by itself it would cause spin precession in the horizontal plane. $\omega_{a} / 2 \pi$ is referred to as the " $(g-2)$ " frequency. If there is an EDM of magnitude $d=\eta e \hbar / 4 m c \approx \eta \times 4.7 \times 10^{-14} \mathrm{e} \cdot \mathrm{cm}$, there will be an additional precession angular frequency

$$
\vec{\omega}_{e}=\frac{\eta}{2} \frac{e}{m} \vec{\beta} \times \vec{B}
$$

about the direction of $\vec{E}^{*}$, that is in the radial direction with respect to the orbit [8]. The vector combination of $\vec{\omega}_{a}$ (vertical) and $\vec{\omega}_{e}$ (radial) tilts the precession plane out of the horizontal plane, leading to a vertical component of spin which oscillates at the frequency $\omega=\sqrt{\omega_{a}^{2}+\omega_{e}^{2}}$ with an amplitude proportional to the EDM. The decay electron direction is correlated with the spin direction; therefore the decay electrons acquire a small oscillating vertical component of momentum. A search for this oscillation during the CERN $(g-2)$ experiment [12] led to the current muon EDM limit [ $[$ ] which corresponds to $\omega_{e} / \omega_{a} \leq 10^{-2}$.

This method has been used during the muon $(g-2)$ experiment at Brookhaven [13, but is limited by serious systematic effects. First, because of the $(g-2)$ rotation, the EDM effectively acts for only one quarter of the $(g-2)$ period, thereby reducing the EDM signal. Also the two extremes of the vertical oscillation occur when the 
muon spin is aligned radially inwards and outwards. In these two extremes the decay electrons, whose up/down asymmetries are to be compared, follow rather different tracks through the magnetic field. In one case the majority are emitted radially inwards and take a short path to the detectors; while in the other case they are emitted predominantly outwards and reach the detectors after a longer track with more opportunity to spread vertically or to be bent by stray radial magnetic fields. The horizontal $(g-2)$ precession thus interferes with attempts to observe the vertical precession due to the EDM.

The new technique is to cancel the $(g-2)$ precession $\vec{\omega}_{a}$ so that the radially directed $\vec{\omega}_{e}$ can operate by itself. For a particle whose spin is initially polarized along its momentum direction, the spin will rotate about the radial direction, acquiring a vertical component, so that the angle between the spin and the horizontal (orbit) plane increases from zero linearly with time. Instead of a small vertical spin component oscillating above and below the plane, we now have a vertical spin component which can, by comparison, become quite large with time, thereby greatly enhancing the EDM signal. The cancellation can be achieved by applying a strong radial electric field $\vec{E}$ to the orbit. The expression for the angular velocity vector of the $(g-2)$ precession [10, 11] in the presence of an $\vec{E}$ as well as a $\vec{B}$ field is, with $\vec{\beta} \cdot \vec{E}=\vec{\beta} \cdot \vec{B}=0$,

$$
\vec{\omega}_{a}=\frac{e}{m}\left[a \vec{B}+\left(\frac{1}{\beta^{2} \gamma^{2}}-a\right) \vec{\beta} \times \vec{E} / c\right]
$$

valid for both fermions and bosons. The latest muon $(g-2)$ experiments [12, 13] run at the "magic" momentum of $3.1 \mathrm{GeV} / c$ with $\gamma \approx 29.3$ where the second term of equation 2 vanishes and $\vec{\omega}_{a}=a(e \vec{B} / m)$. For the dedicated EDM experiment we are proposing to use muons with momentum below the "magic" momentum value. If $1 /\left(\beta^{2} \gamma^{2}\right) \gg a$ and the electric field is adjusted to

$$
E=E_{0} \approx a B c \beta \gamma^{2}
$$

$\omega_{a}$ can be reduced to zero (see also 14]). The correct value can be set in the laboratory by monitoring the cancellation of the $(g-2)$ precession with electron detectors on the inside of the ring. Then $\vec{\omega}_{e}$ in equation (II) will have its full effect, moving the spin steadily out of the horizontal plane. The vertical asymmetry can be observed with detectors, located above and below the orbit, to measure the EDM without the systematic errors mentioned above.

To obtain the best accuracy it is desirable to use a high magnetic field and high energy muons which live longer. But equation (3) shows that this would require impractically large electric fields. The parameters of a possible experiment are shown in Table I.

Table I. Parameters of a possible muon EDM experiment.

\begin{tabular}{|c|c|c|c|c|c|}
\hline $\mathrm{E}$ & Aperture & $\mathrm{B}$ & $\mathrm{p}$ & $\gamma \tau$ & $\mathrm{R}$ \\
\hline $2 \mathrm{MV} / \mathrm{m}$ & $0.1 \mathrm{~m}$ & $0.25 \mathrm{~T}$ & $0.5 \mathrm{GeV} / \mathrm{c}$ & $11 \mu \mathrm{s}$ & $7 \mathrm{~m}$ \\
\hline
\end{tabular}

The uncertainty in $\eta$ is

$$
\sigma_{\eta}=\frac{\sqrt{2}}{\gamma \tau(e / m) \beta B A P \sqrt{N}}
$$

where $A$ is the vertical asymmetry of the detected electrons for $100 \%$ muon beam polarizarion and $P$ is the actual muon beam polarization. $\mathrm{N}$ is the total number of detected electrons, and $\tau$ is the muon lifetime at rest.

For example, to reach the sensitivity of $10^{-24} \mathrm{e} \cdot \mathrm{cm}$ in the EDM corresponding to $\omega_{e} / \omega_{a}=10^{-8}$, the vertical spin angle to be measured after 3 dilated lifetimes $(33 \mu \mathrm{s})$ would be $\sim 50 \mathrm{nR}$, generating a counting asymmetry of $10^{-8}$ and requiring about $4 \times 10^{16}$ registered events, assuming $A=0.3$ and $P=0.5$, i.e. $N P^{2}=10^{16}$. Reaching $10^{-24} \mathrm{e} \cdot \mathrm{cm}$ would require a high intensity muon source plus a storage ring of large acceptance. The muon EDM collaboration has submitted a letter of intent to JPARC [15] where the requisite muon beam line has been proposed.

In practice detectors, called $(g-2)$ detectors, would be set up to monitor the $(g-2)$ precession (horizontal spin motion). Other detectors, above and below the orbit called EDM detectors, would be set up to measure the vertical spin motion. With the electric field set to some value below $E_{0}$ one can observe the $(g-2)$ precession and determine its precession plane. As $E$ approaches $E_{0}$ the $(g-2)$ frequency $\omega_{a} / 2 \pi$ will gradually decrease. At the same time the EDM signal should have the same period as $(g-2)$ but its amplitude should grow. When the $(g-2)$ motion is cancelled the EDM signal should grow linearly with time. In principle $\omega_{e}$ could make the spin turn several times in the vertical plane. But if the EDM is small, as expected, one will only observe the beginning of the first oscillation, that is a slow linear rise.

Any storage ring must have horizontal and vertical focusing (quadrupoles or magnetic gradients) to keep the particles in orbit and the particles will in general make betatron oscillations about the equilibrium orbit which will not necessarily be exactly flat or in one plane. A number of imperfections in the magnetic or electric fields would make the spin move out of the plane of the orbit even though the EDM is zero, giving rise to a false EDM signal. In the discussion "the horizontal plane" means the plane of the orbit, while "vertical" and subscripts "V" refer to components normal to the plane of the orbit. The following imperfections have been considered:

1. Vertical corrugations of the orbit due to a radial magnetic field $B_{r}$.

2. The plane of the radial electric field does not coincide with the plane defined by the magnetic field (called the "magnetic plane"), that is $E_{\mathrm{v}} \neq 0$ although the electric field is perfectly in a single plane so $\left\langle E_{\mathrm{v}}\right\rangle=0$ with the brackets \langle\rangle indicating the average over the orbit.

3. The electric field is not in one plane, $\left\langle E_{\mathrm{v}}\right\rangle \neq 0$. 
4. Local orbit distortions near the detectors simulate detector rotation around the beam direction, so small residual $(g-2)$ precession ("horizontal") has a component in the "vertical" direction looking like a false EDM.

5. Change of up detector response relative to down detector response during the muon storage time.

6. Azimuthal components $B_{\theta}$ of the magnetic field parallel to the momentum vector $\vec{p}$. Although $\left\langle B_{\theta}\right\rangle=0$ if there is no electric current through the orbit, higher harmonics of the azimuthal B-field could be significant.

We will discuss these effects in turn. In any ring structure with magnetic and/or electric fields, for each particle momentum there exists a closed orbit; the particle repeats this track perfectly from turn to turn. Other particles, starting at different transverse positions or transverse angles, oscillate about the closed orbit. To define the plane of the closed orbit, split it up into many small equal sections, each with its local angular velocity vector $\vec{\omega}$ and find the average value $\langle\vec{\omega}\rangle$. The orbit plane is defined as the plane perpendicular to $\langle\vec{\omega}\rangle$; on average the momentum vector rotates in this plane but may oscillate above and below it. With only a magnetic field, the orbit plane will therefore be defined by the average direction of $\vec{B}$. The radial electric field may not lie exactly in this plane. In this case the orbit plane will change when the electric field is applied.

Since we are interested in the spin direction relative to the momentum vector [10], we consider the electric and magnetic field components $\vec{E}^{*}$ and $\vec{B}^{*}$ in the rest frame of the particle circulating in the orbit plane [10]. For the closed orbit to be stable vertically, the mean vertical force in the lab frame

$$
\left\langle E_{\mathrm{v}}+\beta B_{r}\right\rangle=0 .
$$

Transforming to the rest frame one finds $\left\langle E_{\mathrm{v}}^{*}\right\rangle=0$, not unexpectedly because in the rest frame it is $\vec{E}^{*}$ that moves the orbit while $B^{*}$ generates no force.

$B_{r}^{*}$ rotates the spin out of the orbit plane when the EDM is zero. Using Eq. (5)

$$
\left\langle B_{r}^{*}\right\rangle=\left\langle\gamma B_{r}+\beta \gamma E_{\mathrm{v}}\right\rangle=-\left\langle E_{\mathrm{v}} / \beta \gamma\right\rangle .
$$

It follows that with no electric field there is no false EDM whatever the shape of the orbit (error 1). If the radial electric field is exactly in one plane so that $\left\langle E_{\mathrm{v}}\right\rangle=$ 0 then $\left\langle B_{r}^{*}\right\rangle$ is zero and there is again no false EDM, (error $2)$.

A further effect of electric field misalignment is that $\vec{\beta} \times \vec{E}$ is not parallel to $\vec{B}$ so that when (3) is satisfied there is a net horizontal angular velocity $\vec{\omega}_{r}$ acting on the spin. However, if this is radially inwards on one side of the ring, it will be radially outwards on the other, generating a small vertical spin oscillation which does not accumulate from turn to turn as long as the $(g-2)$ precession is zero: no false EDM. If there is a residual $(g-2)$ precession and a radial electric field is present, it is possible that a radial spin component can be transformed into a vertical component. This is the case when the radial electric field is not exactly orthogonal to the magnetic field. A single detector at a specific azimuthal location will observe a small EDM like signal that has the opposite sign at a detector located $180^{\circ}$ apart. The effect is proportional to the misalignment of the electric and magnetic fields from orthogonality and it goes to zero when the detector signals from all azimuthal locations are summed.

If the radial electric field is not precisely in a plane (error 3) there will be a net vertical electric field $\left\langle E_{\mathrm{v}}\right\rangle \neq 0$. This will move the orbit until $\left\langle B_{r}^{*}\right\rangle$ satisfies Eq. [6] and this will precess the spin out of the plane generating a false EDM. Every precaution must be taken to minimize this effect but fortunately it is cancelled by alternatively injecting the particles clockwise $(\mathrm{CW})$ and counter-clockwise (CCW) and subtracting the counts in the detectors. This requires discussion of the signs of the real and false EDM signals for $\mu^{+}$and $\mu^{-}$in each case. The following equations indicate the signs (not magnitudes) of the real and false EDM angular velocities $\vec{\omega}_{e}$ and $\vec{\omega}_{F}$ :

$$
\begin{aligned}
\vec{\omega}_{e} & \propto \vec{\sigma} \times[\vec{d} \times(\vec{p} \times \vec{B})] \\
\vec{\omega}_{F} & \propto \vec{\sigma} \times\left[\vec{\mu} \times\left(\vec{p} \times \vec{E}_{\mathrm{v}}\right)\right]
\end{aligned}
$$

where $\vec{\sigma}$ represents the spin vector. If there is a finite EDM $\vec{d}$, the CPT theorem requires $\vec{d} \cdot \vec{\sigma}$ to change sign going from $\mu^{+}$to $\mu^{-}$. In Table II we show the truth table for the four different configurations $\mu^{+} / \mu^{-}$combined with the orbit directions $\mathrm{CW} / \mathrm{CCW}$ listing the variables in the order $\vec{p}, \vec{\sigma}, \vec{\mu}, \vec{d}, \vec{B}, \vec{E}_{\mathrm{v}}$. We are displaying the situation at a fixed point in the ring, assuming that the muons come from pion decay in the backward direction and we arbitrarily make all variables positive for the reference case $\left(\mu^{+}, \mathrm{CW}\right)$.

Table II. Truth table for the four different configurations $\mu^{+} / \mu^{-}$combined with the orbit directions CW/CCW.

\begin{tabular}{|c|l|c|}
\hline & CW & CCW \\
\hline Particle & $\vec{p}, \vec{\sigma}, \vec{\mu}, \vec{d}, \vec{B}, \vec{E}_{\mathrm{v}}$ & $\vec{p}, \vec{\sigma}, \vec{\mu}, \vec{d}, \vec{B}, \vec{E}_{\mathrm{v}}$ \\
\hline$\mu^{+}$ &,,,,,++++++ &,,,,,-----+ \\
\hline$\mu^{-}$ &,,,,,+-++-- &,,,,,-+--+- \\
\hline
\end{tabular}

If the muons are emitted backward in the decay of pions in flight, the majority of decay electrons initially go forward (in the direction of $\vec{p}$ ), so the observed asymmetry obeys:

$$
\vec{A}_{e, F} \propto \vec{p} \times \vec{\omega}_{e, F} .
$$

Applying equations [7, [8 and 9 the results for real EDM asymmetry $A_{e}$ and false EDM asymmetry $A_{F}$ are listed in Table III. 
Table III. The results for real EDM asymmetry $A_{e}$ and false EDM asymmetry $A_{F}$, applying Eqs. (7)-(9).

\begin{tabular}{|c|c|c|}
\hline & CW & CCW \\
\hline Particle & $A_{e}, A_{F}$ & $A_{e}, A_{F}$ \\
\hline$\mu^{+}$ &,++ &,-+ \\
\hline$\mu^{-}$ &,++ &,-+ \\
\hline
\end{tabular}

We see that the real EDM signal changes sign when the direction of rotation in the ring is reversed, while the false EDM due to the out-of-plane electric field remains the same. So this error may be cancelled by changing from $\mathrm{CW}$ to $\mathrm{CCW}$ if all other factors can be held the same.

If the electric field is misaligned but in a plane (error 2) the orbit plane will change when the electric field is applied, so the detectors, set to respond only to vertical spin components will include small contribution from horizontal spin. This will however be opposite on opposite sides of the ring and so will largely cancel. Error 4 has a similar effect.

The response of upper and lower detectors may change with time (error 5), so that a false asymmetry develops during each muon storage cycle. Such effects can be caused by unequal detector responses to the changing counting rates or transients in the system triggered at injection time. This effect should remain the same when muons are injected $\mathrm{CW}$ and $\mathrm{CCW}$ while the real EDM asymmetry changes sign.

It might be supposed that the electric and magnetic fields could be applied to separate sections of the orbit, with the result that the spin makes small to and fro movements about the vertical axis but the net $(g-2)$ precession is zero over one turn. While this would fulfill the main requirement, some misalignment errors would not be perfectly cancelled. For example, a harmonic of the azimuthal field $B_{\theta}$ (error 6) would cause the spin to oscillate about the horizontal axis parallel to $\vec{p}$. Because rotations do not commute, the combination with the $(g-2)$ oscillation would generate a net rotation about the radial axis, leading to a false EDM. This is an example of Berry's phase [16]. Similarly, a misalignment of the electric field (error 2) would generate an oscillating radial angular velocity $\omega_{r}$ as explained above. Combined with the $(g-2)$ oscillation this would give rise to a false EDM. To minimize these effects, the electric and magnetic fields must be located at the same place. This error is also canceled by injecting CW and CCW.

Further tests can be made by injecting muons with the opposite longitudinal polarization coming from pion decay in the forward direction. In this case the maximum decay electron intensity is directed backwards and all asymmetries are reversed. But a false asymmetry due to detector effects (error 5) should remain the same.

Therefore, all false signals, unlike the EDM signal, will be cancelled by $\mathrm{CW}$ and CCW beam injection and by summing up the counts of all the detectors.

The method can be applied to other particles or atoms provided [17] that the $g$-factor is not too far from 2 so the $(g-2)$ precession can be cancelled by an accessible electric field, for example the deuteron. Since the deuteron is stable, another scheme must be utilized to track the deuteron spin. This would most likely involve the use of an internal target in the ring. One possible target is hydrogen gas as elastic $\mathrm{d}+\mathrm{p}$ scattering is sensitive to all of the polarization moments of the deuteron beam [18].

In conclusion, we have presented a new method which improves the sensitivity of EDM search for charged particles in storage rings by several orders of magnitude. It is achieved by applying an external radial electric field which cancels all sources of spin precession except that due to a non-zero EDM. It is most applicable to particles with a small anomalous magnetic moment.

This work was supported in part by the U.S. Department of Energy. We wish to thank the members of the EDM collaboration for comments and criticism on the manuscript.
[1] L.Landau, Nucl.Phys. 3, 127 (1957).

[2] M. Pospelov and I.B. Khriplovich, Sov. J. Nucl. Phys. 53 638 (1991).

[3] A. Pilaftsis, Nucl. Phys. B644, 263 (2002); J.L. Feng, K.T. Matchev, and Yael Shadmi, Nucl. Phys. B613, 366 (2001); J.R. Ellis et al., Phys. Lett. B528 86 (2002); A. Romanino and A. Strumia, Nucl. Phys. B622, 73 (2002).

[4] K.S. Babu, B. Dutta, and R.N. Mohapatra, Phys. Rev. Lett. 85, 5064 (2000).

[5] B.C. Regan et al., Phys. Rev. Lett. 88, 071805 (2002).

[6] J. Bailey, et al., J. Phys. G4, 345 (1978).

[7] L.I. Schiff, Phys.Rev. 132, 2194 (1963).

[8] R.L. Garwin and L. Lederman, Nuovo Cimento 11, 776 (1959).

[9] P.G.H. Sandars, Contemporary Physics 42 (2):97 (2001).

[10] V. Bargmann, L. Michel, and V.L. Telegdi, Phys. Rev.
Lett. 2, 435 (1959).

[11] J.D. Jackson, "Classical Electrodynamics", p. 559, $2^{\text {nd }}$ ed., Ed. John Wiley and Sons, New York, 1975.

[12] J. Bailey et al., Nucl. Phys. B150, l (1979).

[13] G. W. Bennett et al., Phys. Rev. Lett. 92, 161802 (2004).

[14] D.F. Nelson et al., Phys. Rev. Lett. 2, 492 (1959), (was discovered by the authors after the current paper was accepted for publication).

[15] M. Aoki et al, J-PARC LOI: "Search for a Muon EDM at the $10^{-24} \mathrm{e} \cdot \mathrm{cm}$ Level", http://www-ps.kek.jp/jhf-np/LOIlist/pdf/L22.pdf J-PARC: Japan Proton Accelerator Research Complex, http://j-parc.jp

[16] M.V. Berry, Proc. Roy. Soc. London A392, 451 (1984).

[17] I.B. Khriplovich, Phys. Lett. B444, 98 (1998).

[18] W. Glöckle, H. Witała, D. Hüber, H. Kamada, and J. 
Golak, Phys. Rep. 274, 107 (1996). 